CAHIERS DE

NARRATOLOGIE

\section{Cahiers de Narratologie}

Analyse et théorie narratives

$10.2 \mid 2001$

La voix narrative

\title{
La « voix » des contes de fées dans La reina de las nieves de Carmen Martín Gaite
}

\section{Philippe Merlo}

\section{(2) OpenEdition}

1 Journals

Édition électronique

URL : http://journals.openedition.org/narratologie/10227

DOI : 10.4000/narratologie.10227

ISSN : 1765-307X

Éditeur

LIRCES

Édition imprimée

Date de publication : 1 janvier 2001

Pagination : 253-267

ISBN : 2914561032

ISSN : 0993-8516

Référence électronique

Philippe Merlo, "La « voix » des contes de fées dans La reina de las nieves de Carmen Martín Gaite », Cahiers de Narratologie [En ligne], 10.2 | 2001, mis en ligne le 01 janvier 2001, consulté le 11 juin 2020 URL : http://journals.openedition.org/narratologie/10227 ; DOI : https://doi.org/10.4000/narratologie 10227 


\title{
LA « VOIX » DES CONTES DE FÉES DANS LA REINA DE LAS NIEVES DE CARMEN MARTÍN GAITE
}

\author{
Philippe MERLO \\ Université de Provence, Aix-Marseille I \\ GRIMIA, Université Lumière-Lyon 2
}

Parler de « la voix narrative » au singulier peut sembler, dans un premier temps, assez limitatif si l'on ne considère pas cette voix comme le singulier qui cache et évoque un pluriel et par conséquent une polyphonie. Dès le départ, il faut donc se mettre d'accord sur ce que l'on entend par voix narrative. Est-ce, comme le précise Gérard Genette, non pas seulement celui qui accomplit ou subit l'action, " mais aussi celui (le même ou un autre) qui la rapporte, et éventuellement toux ceux qui participent, fût-ce passivement, à cette activité narrative' ${ }^{1}$ ? Ou, faut-il aller encore plus avant dans cette démarche en considérant aussi comme voix narratives toutes celles qui peuvent intervenir dans et autour d'un récit : celles des différents narrateurs internes au récit - narrateurs homo et hétérodiégétiques - et celles des personnes externes au récit que sont les lecteurs. On peut penser qu'il existe aussi une troisième catégorie qui regroupe les personnages qui, dans les romans, n'ont pas le statut de narrateur mais dont un narrateur rapporte les propos, ce qui sous-entend que ces personnages furent, à un moment donné du passé, narrateurs à leur tour.

La voix narrative renvoie à un narrateur qui est celui qui fait une narration, c'est-à-dire, comme le précise le Littré, un " récit historique oratoire ou poétique " ou bien un "simple récit fait en conversation ». Dans le premier cas, l'accent est mis sur le genre et sur une certaine rhétorique tandis que la deuxième acception insiste sur le dialogue et la transmission d'un savoir par l'oralité. La question qui se pose alors est au

${ }^{1}$ Gérard GENETTE, Figures III, Paris, Seuil, Poétique, 1972, p. 226. 
moins double : comment s'imbriquent les différents niveaux de voix narratives à l'intérieur d'un même roman et comment l'oralité que suggère la narration est intégrée au récit écrit ?

Pour mettre en évidence ces différents niveaux de voix narratives, nous avons choisi de partir de l'analyse d'un roman espagnol contemporain assez récent de Carmen Martín Gaite qui s'intitule La Reina de las Nieves (1994). Comme l'indique le titre choisi par la romancière, le lecteur se trouve pour ainsi dire face à une citation palempsestique au sens genettien du terme qui renvoie au conte d'Hans Christian Andersen La Reine des Neiges. Le roman de Martín Gaite raconte en grande partie les souvenirs d'enfance du héros, Leonardo, qui, devenu adulte, se souvient de son passé pendant lequel sa grand-mère a joué un rôle fondamental dans son éducation en lui racontant des contes de fées. Nous tenterons donc de répondre à la double problématique mise en évidence en précisant tout d'abord quels sont les multiples niveaux narratifs mis en présence pour mieux en analyser les échanges, les interférences, les fusions, voire les confusions. Finalement, nous montrerons comment, dans le roman de Carmen Martín Gaite, le passage de la voix orale à la voix écrite se réalise en empruntant certains moyens plutôt que d'autres.

La Reina se divise en trois parties clairement identifiées comme telles par l'écrivain : "Primera parte », "Segunda parte (De los cuadernos de Leonardo)» et «Tercera parte»: le narrateur des première et troisième partie semble identique - narrateur hétérodiégétique à la troisième personne du singulier - tandis que la narration de la deuxième partie incombe à un narrateur intra homodiégétique, Leonardo qui emploi le " yo » et qui narrateur-adulte raconte ses souvenirs d'enfance. La narration aux allures autobiographiques se trouve ainsi enchâssée par une narration qui aborde les mêmes aspects thématiques mais perçus et racontés à partir d'une extériorité qui, conjuguée à la narration à la première personne du singulier, propose au destinataire qu'est le lecteur, une vision relativement plus objective. Par ailleurs, la voix du récit de la deuxième partie renvoie non pas à un discours dit mais 
plutôt écrit sur des cahiers, mêlant ainsi étroitement oralité et écriture : deux axes fondamentaux pour la compréhension de toute l'œuvre.

Tout le roman de La Reina fait apparaître une multitude de voix non seulement liées à des narrateurs mais aussi à certains personnages dont la fonction première est de raconter des histoires. Ainsi en est-il pour la grand-mère du héros, nous l'avons dit, mais aussi d'autres personnages tels que le vieil homme qui raconte l'histoire du dernier torrero à occuper le phare qui se trouve tout près de la Quinta Blanca où a vécu Leonardo (51). La description du vieil homme n'est pas sans nous en rappeler une autre, celle du héros de Le vieil homme et la mer d'Ernest Hemingway. En outre, les points de rapprochements entre les deux romans sont multiples. Mais, ce qui doit être souligné ici est la mise en place d'un groupe de personnages - les vieilles personnes - qui joue le rôle de réservoir de souvenirs, une sorte de mémoire vivante du passé dont ils sont à la fois les dépositaires et les transmetteurs. Le roman évoque très souvent la place centrale que prennent ces personnages au moment où ils commencent à être la voix du passé. C'est alors toute une mise en scène de la voix qui est convoquée : description du cercle formé par les auditeurs qui entourent le narrateur, silence autour de la voix qui parle, convivialité et détente. La scène semble alors ne plus se dérouler dans le temps linéaire de la narration livresque, mais bien dans une atemporalité propre au conte qui serait semblable à celui du mythe, comme le précise Mircéa Éliade qui, par ailleurs, est cité au moins par deux fois dans le roman $(215,226)$. Le roman met ainsi en place un troisième niveau de voix narrative : outre celui du narrateur hétérodiégétique de la première et troisième partie, outre celui du narrateur homodiégétique de la deuxième partie, il apparaît un troisième narrateur présent dans les trois parties et qui prend en charge la narration des contes de fées.

Comme nous avons pu le mettre en évidence dès l'introduction, le roman reprend en grande partie des passages du conte d'Andersen. Cet aspect générique accentue la place accordée à l'oralité des contes de fées, à leur transmission d'une génération à une autre par les voix des narrateurs et narratrices qui rapportent les histoires. Comme le précise 
Michèle Simonsen dans son étude sur Le conte populaire : «[...] le mot conte, de nos jours encore, a un sens très clair. IL est lié d'emblée à l'acte de conter, donc à l'oralité, et à la fictivité : c'est un récit qui n'est pas 'vrai'. ${ }^{2}$ » Il évoque de plus un «monde traditionnel, 'la relative stabilité d'un monde plus clos'3. " Dans La Reina, le conteur par excellence est incarné par le personnage de la grand-mère, une des figures de narrateur les plus récurrentes des contes enfantins que Michèle Simonsen mettrait dans la catégorie des « spécialistes peu nombreux, dont les goûts, la mémoire, le talent et les hasards de l'existence ont fait des conteurs ${ }^{4}$. " Son apparition est systématiquement liée à la narration d'une histoire ou d'un conte, comme le prouve la répétition par quatre fois sur une seule page (p. 81) du verbe «decir » qui introduit les propos de la grand-mère. Le roman, par le jeu de l'intertextualité, propose alors un quatrième niveau de narration, distinct des niveaux précédents, qui renvoie à la fois vers un autre temps - celui de l'enfance du narrateur adulte -, vers un autre genre - celui du conte -, et vers une autre voix qui est celle de la grand-mère qui se fond dans les contes. En bien des aspects, les quatre niveaux narratifs sont identiques à ceux mis en place par un autre romancier espagnol - Juan Marsé dans son œuvre Si te dicen que caí écrite et publiée bien avant La Reina. Les niveaux de voix narratives se complexifient et cela d'autant plus que l'oralité des contes de fées amène le narrateur à proposer à son auditoire une version à chaque fois différente de la version antérieure, comme pour mieux mettre en évidence la variété mais aussi la fragilité qui caractérisent la transmission orale. Le conte, comme tout genre transmis oralement, comprend ce que Michèle Simonsen appelle "des éléments, stables, te des éléments fluides, plus mobiles. Ces derniers peuvent varier d'une narration à l'autre, chez le même conteur, qui improvise chaque fois un peu, à

2 Michèle SIMONSEN, Le conte populaire, Paris, PUF, 1984, p. 15.

3 Marie-Louise TENÈZE, "Introduction à l'étude de la littérature orale : le conte " in Annales, Économies, Sociétés, Civilisations, septoct 1979 citée par Michèle Simonsen.

${ }^{4}$ Michèle SIMONSEN, op. cit., p. 38. 
partir de divers procédés mnémotechniques ${ }^{5}$ ", comme nous pouvons nous en rendre compte dans l'exemple qui suit :

Siempre que volvía a hacer la narración de aquel encuentro, enriquecida cada vez con nuevos detalles, y ramificada por las múltiples divagaciones que el tema iba concitando en su memoria, volvía a sacar Rosa de la faltriquera aquel pañuelo de batista con las inciales E.V.G. bordadas en una esquina para secarse con él las lágrimas que van a engrosar los ríos cuyas aguas nunca vuelven. (21-22)

On soulignera bien sûr le "volver a » qui insiste sur la répétition de l'histoire, le retour d'une narration déjà faite, mais aussi et surtout sur l'accumulation des expressions qui traduisent à la fois la différence et la nouveauté entre une version et une autre de la même histoire : "enriquecida", " nuevos detalles », « ramificada », « múltiples divagaciones ». Le roman insiste d'ailleurs sur cette oralité, car même lorsque la grand-mère semble lire le conte à son petit-fils à partir d'un livre, en fait elle raconte l'histoire oralement car elle la connaît par cœur (105). En agissant ainsi, la grand-mère, tout comme son petit-fils, réactualisent en permanence un acte fondateur et pénètrent dans un vaste ensemble de transmission de la mémoire par l'oralité et par abolition du temps linéaire, s'intégrant alors dans un temps mythique tel que le définit Mircea Eliade pour qui « il en est de même pour toutes les répétitions, c'est-à-dire toutes les imitations des archétypes; par cette imitation, l'homme est projeté à l'époque mythique où les archétypes ont été pour la première fois révélés. [...] il y a abolition implicite du temps profane, de la durée, de l' "histoire », et celui qui reproduit le geste exemplaire se trouve ainsi transposé dans époque mythique où a eu lieu la révélation de ce geste exemplaire ${ }^{6}$. »

*

La toute première des relations qui unit les différentes voix narratives des deux niveaux génériques est d'ordre

5 Ibid., p. 40.

${ }^{6}$ Mircea ElIADE, Le mythe de l'éternel retour, Paris, Gallimard, Idées, 1969, p. 49-50. 
purement linguistique. En effet, ce sont les mots propres au conte et à ses narrateurs internes qui viennent réapparaître dans les propos tenus par les personnages et les narrateurs du récit romanesque. Nous assistons à une véritable contamination verbale du roman par le conte à travers les « trabalenguas », c'est-à-dire les allitérations, les jeux sur les sonorités, comme dans l'exemple suivant :

Y me asaltaron de nuevo aquellas cantinelas surrealistas con pregunta implícita que la abuela me proponía, a modo de jeroglífico : " De codín de codán, de la vera vera van, de la bodega al desván, de la alcoba hasta el zaguán, de la sala a la cocina, ¿cuántos dedos hay encima? » (95)

On voit bien que c'est la grand-mère qui prête à la fois sa voix aux contes de fées et qui, présente dans le roman en tant que personnage, sert de lien entre les deux récits. Le narrateur adulte du récit romanesque rapporte les propos tenus par la grand-mère, narratrice des contes. Mais le narrateur adulte ne se limite pas à un rôle de rapporteur, il va faire siennes ces paroles pour les intégrer à sa propre narration et aux questionnements propres à l'adulte, comme lorsqu'il se souvient d'un des échanges qu'il a pu avoir avec sa grandmère (80). Ce conte qui est si difficile à comprendre n'est rien d'autre que la vie du narrateur adulte qui ne sait quelle est la voie-x qu'il doit suivre: celle suggérée par les propos énigmatiques de sa grand-mère dans sa jeunesse qui laisse planer une triple interrogation - «quién pasó », "quién parecía », " quién se escondió »- ou celle du narrateur adulte qui se trouve dans l'impossibilité de choisir? Leonardo va encore plus loin puisqu'il parvient à « historiciser» dans le récit romanesque qui est le sien les «trabalenguas » en les mélangeant aux réalités spatio-temporelles qui furent son quotidien quand il était enfant, mais aussi à sa réalité de narrateur adulte. Il s'agit de l'introduction de la Quinta Blanca - lieu où Leonardo vécu avec sa grand-mère - dans les «trabalenguas » des contes, donc à l'époque de l'enfance, mais aussi mélangée à la narration prise en charge par l'adulte :

Cuando se alejaba de mí se reía, cuando se acercaba no, se iba poniendo seria y daba un poco de miedo, se le quedaba la cara tan parada como el día que la vi muerta, 
la última vez que pisé la Quinta Blanca, blanca la quinta muerta, blanca la abuela muerta, pero seguía teniendo un poco de risa detrás de la mueca seria, se notaba después de mirarla un rato.

- Deja los acertijos, anda abuela, que no los entiendo. Mejor cuéntame un cuento. (81)

Comme on peut s'en rendre compte à travers cette citation, si le premier paragraphe a pour voix narrative celle de Leonardo adulte, sérieux, qui évoque la mort, le second, qui emploie le style direct, revient à Leonardo enfant qui ne veut pas connaître autre chose que la fiction des contes comme le suggère fortement la tournure pléonastique de "cuéntame un cuento ». Nous assistons alors à une fusion des niveaux temporels et génériques de même qu'à une confusion des voix qui n'a pour d'autre effet que celui de confondre les deux narrateurs qui se cachent derrière un seul et même personnage. Les voix narratives font alors apparaître d'autres thèmes fondamentaux du roman : l'oralité, la mémoire et le dédoublement.

Le roman va favoriser le dédoublement des voix narratives par l'instauration d'éléments qui suggèrent fortement le thème du double, de la dualité. Ainsi en est-il pour l'espace où se déroule l'enfance du narrateur, à savoir la Quinta Blanca et plus particulièrement ses environs :

Eran, no cabía duda, los acantilados del faro, escenario donde se incubaron mis primeras rebeldías, donde di alas a tantos inconcretos anhelos de aventuras y empecé a idealizar, asomado al abismo, el destino de Kay. (121)

La description du phare, des falaises mettent en place une re-présentation, une scénification - « escenario »- de la profondeur abyssale - «acantilados » et bien sûr « abismo »propose une mise en abî(y)me du personnage qui se penche pour mieux se voir, tel Narcisse, mais aussi pour mieux observer son double - sa doublure dirait-on au théâtre - dans le personnage de Kay. L'abîme spacial renvoie tout d'abord à la spécularité identitaire :

Hay emociones que nunca se entierran del todo. Tuve que acudir al recuerdo de Octavio Andrade para tranquilizarme. Se imprimió un pequeño flashback a la película, se disipó la niebla y de aquella casa, que ahora apenas se 
veía, salió un Leonardo Villalba que caminaba con paso seguro hacia su identidad de heredero respetable y a quien no hubiera alterado la mirada torva de ningún taxista. (232)

Dans cet extrait, le narrateur intra homodiégétique qu'est Leonardo adulte évoque sa propre personne sous les traits d' " un Leonardo Villalba » en quête de son identité. La mise en place de cette altérité est renforcée par le regard extérieur que porte le chauffeur de taxi que l'on peut imaginer en train d'assister au dédoublement de personnalité. De plus, l'emploi du verbe "alterar » insiste de par son étymologie alter = autre - sur la création de cette "ipséité » au sens que Ricoeur donne à ce terme ${ }^{7}$. La spécularité identitaire se voit alors doublée à son tour par une spécularité visuelle que suggèrent les nombreuses récurrences du miroir dans l'œuvre. Il ne pouvait en être autrement lorsqu'on se rappelle que l'étymologie de miroir est speculum en latin. Le miroir évoque le thème du dédoublement de la personnalité et, à travers lui, celui d'une voix qui se duplique. Le miroir est toujours concave (289) ou ovale (144), et cette rondeur peut à fois révéler et/ou occulter (289). Quoi qu'il en soit, le miroir entraîne vers une "autre réalité ». Pensons aux nombreuses évocations des miroirs dans les contes de fées, comme celui avec lequel dialogue la reine-marâtre de Blanche-Neige ou ceux qui permettent le passage d'un espace à un autre, d'une dimension vers une autre évoquée par les derniers mots de la citation ci-dessus et où limites, barrières et frontières semblent avoir disparu. Par conséquent, les personnages et le narrateur homodiégétique en particulier ne parviennent plus à faire la différence entre leur vie personnelle et celle des héros des contes. C'est bien ce qui arrive à Leonardo qui se confond avec le personnage de Kay. Une fusion que la répétition du verbe « unir » traduit clairement, de même que l'évocation du héros du conte de fées à côté du « yo » du narrateur :

Y fue entonces cuando intuí cómo la distancia puede unir a dos seres desgraciados de forma más indisociable que la cercanía de sus cuerpos. Nos unía - y nos sigue

7 Paul RICOEUR, Soi-même comme un autre, Paris, Seuil, 1990, p. 11-38. 
uniendo - la desesperación de nuestras respectivas pesquisas divergentes, ella en busca de Kay y yo, sin saberlo, en busca de alguien tan valiente como ella. Nuestras voces se cruzan aún sin encontrarse. (202)

Mais, le plus intéressant, il est vrai, est la dernière phrase de cette citation qui montre que la fusion n'est pas seulement héroïque, mais aussi et surtout narrative : la voix de chacun des héros, du conte et du roman, se croisent, même si elles ne se rencontrent pas encore.

En fait, le personnage de Leonardo, en tant que narrateur homodiégétique, en arrive à se confondre avec les personnages du conte de fée de son enfance que sont Gerda et Kay. Leonardo ressent d'ailleurs très fortement la sensation de se fondre - le terme est employé par le narrateur lui-même avec ces personnages : "Veía sus muslos blancos y desnudos y trataba de acomodar mis pasos al ritmo de los suyos, consciente de que iba a fundirme con ella de un momento a otro » (85). Cette confusion entre les personnalités est essentiellement due aux nombreux points en commun que Leonardo peut avoir avec Gerda ou Kay. Ainsi, Gerda tout comme Leonardo ont tous deux une grand-mère qui leur raconte des histoires, et Leonardo tout comme Kay va recevoir dans son œil un débris du grand miroir qui va les rendre insensibles et surtout amnésiques (p. 103). L'assimilation entre le personnage du roman et le personnage du conte se fait peu à peu par la figure des narrateurs :

«Continúa, pequeño Kay, continúa », le dijo con voz grave pero sin volver el rostro, "hora ya hay que continuar. »

Esta última frase de « ahora ya hay que continuar » no la pronunció nunca la persona alta vestida de blanco, ni tampoco la decía la abuela ; se la añadía yo mentalmente al relato con inmediata espontaneidad. Porque la verdad era que a partir de ese punto ya experimentaba la complicidad con el mal. (107-108)

Alors que la première phrase en style direct semble directement extraite du conte de fées comme le suggère l'emploi des guillemets et la mention de la « voix grave », en fait il n'en est rien, puisque c'est Leonardo lui-même qui prononce ces quelques mots en s'adressant directement à Kay, comme s'il 
était lui-même un personnage du conte. Le conte s'est littéralement introduit dans le personnage et fait partie intégrante de sa personnalité. Le rapprochement entre les différents individus débouche sur une fusion, voire une confusion des voix narratives qui appartiennent d'un côté aux personnages de la fiction première qu'est le roman et, d'un autre, aux personnages de la fiction seconde que sont les contes. Ainsi, la voix de Kay se retrouve dans la bouche du narrateur qui exprime les pensées du personnage féerique : "Ahora ya hay que seguir », recitaba Kay en mi interior, dando diente con diente » (118).

En outre, on remarquera qu'à partir du moment où le narrateur enfant s'assimile aux personnages du roman, à Kay ou à Gerda, il passe de la première personne du singulier à la première personne du pluriel "nosotros », personne grammaticale que les deux enfants du conte utilisent lorsqu'ils parlent de leurs aventures. Leonardo s'inclut donc parfaitement au groupe formé par les deux enfants par le truchement du pronom personnel sujet $(117,127)$. Par ailleurs, l'emploi de cette personne se produit après avoir évoqué dans sa presque totalité le conte de la Reine des Neiges, lors de la séquence 2-IV, comme si l'évocation et l'audition du conte étaient une condition sine qua non à la fusion des voix. Cette confusion a pour conséquence un malaise, un vertige qui provient de la confusion, voire la fusion, entre la réalité et la fiction. Pour tenter de mieux vivre, voire de gommer ce malaise, le narrateur ressent alors le besoin de changer de voix et de passer de la voix de l'oralité à celle de l'écriture. Nous nous situons alors dans le troisième des paramètres principaux du contage qui selon Michèle Simonsen est le répertoire qu'elle définit comme étant "une certaine correspondance entre le type d'institution de transfert et les genres narratifs qui y sont pratiqués ${ }^{8}$. »

Bien vite, le narrateur ressent la nécessité de mettre par écrit les différentes histoires qu'on lui a racontées et qu'il a entendues. Les personnages entre eux ressentent tous ce

8 Michèle SimONSEN, op. cit., p. 36. 
besoin et s'engagent mutuellement à passer de l'oralité à l'écriture. Leur voix se matérialise alors sur le papier par l'écriture (292). Cette écriture de l'oral peut déjà s'observer dans deux cas où le narrateur adulte sert de médium. Tout d'abord, lorsqu'il décide d'écriture dans son journal ses impressions et ses réflexions, ce qui correspond à la deuxième partie du roman qui, rappelons-le, a pour sous-titre « De los cuadernos de Leonardo ». Puis, lorsque Leonardo reprend, dans ses cahiers, des passages entiers du conte d'Andersen. Mais, le passage à l'écriture n'est pas immédiat. Avant d'en arriver à ce stade ultime, le narrateur a besoin de visualiser ce qu'il a entendu en le dessinant $(97-98,175)$. L'illustration devient alors la première étape vers la forme écriture sur le papier.

La voix orale se transforme peu en peu en voix écrite qui sera récupérée par le personnage qui a écouté. D'auditeur, Leonardo, se transforme en narrateur et transmet ainsi le relais à d'autres ou, pour reprendre la terminologie employée par Simonsen, de transmetteur passif, Leonardo, devient un transmettreur actif ${ }^{9}$. Par ailleurs, on remarquera en se mettant à dessiner, Leonardo ne fait que répéter une action que l'on retrouve dans le conte puisque Gerda, dans la troisième histoire du conte qui s'intitule «Le jardin fleuri de la femme qui savait exercer la magie ", peint et illustre la multitude de fleurs qui l'entoure. Cependant, tout comme la jeune fille ne parvient pas à dessiner la rose car la sorcière a tout fait pour l'éradiquer de son jardin afin de ne pas raviver chez Gerda le souvenir de Kay, Leonardo, ne parvient pas à dessiner un élément essentiel et non des moindres puisqu'il s'agit de la Reine des Neiges elle-même (98). Dans les deux cas, qu'il s'agisse de la rose ou du personnage féerique, nous nous trouvons face à des éléments déclencheurs de souvenirs qui, absents, empêchent toute évocation du passé et donc toute possibilité de poursuivre la quête entreprise : celle de retrouver Kay pour Gerda ; celle de renouer avec son passé pour mieux affronter le futur pour Leonardo. Les voix narratives doivent alors se métamorphoser en voix de la mémoire. La deuxième étape nécessaire pour reconstruire le passé, l'histoire individuelle ou collective, est donc de faire inter-

9 Ibid., p. 43. 
venir la mémoire. Les voix du conte sont alors synonymes de voix de l'anamnèse.

Les liens qui existent entre mémoire et écriture sont très étroits comme le souligne André Siganos : "A quelque niveau que l'on se situe, toute écriture est évidemment mémorielle. Entendons par cet adjectif le fait que l'écrivain joue toujours, consciemment ou non, avec le passé de l'Histoire, avec ses propres souvenirs, avec le passé de la langue, celui même du langage avec la culture que le temps a déposée en lui ; quand il ne se trouve pas amené à rapporter de miraculeuses réminiscences ${ }^{10}$. " L'écriture se signale d'emblée sous le signe pressant, voire oppressant de la mémoire, s'appuyant explicitement sur elle pour montrer son extrême dépendance par rapport à un passé qui deviendra, une fois écrit, une mémoire seconde. Les voix de l'oralité proposent un travail à partir des différents niveaux temporels qui jalonnent le roman. Dans un premier temps, elles permettent de renouer avec le passé puisqu'elles émanent des contes de fées de l'enfance du narrateur adulte. La voix du conte est enfouie dans un passé qu'il faut faire resurgir. Elle est à la fois « surmergida » et appartient à un autre narrateur inconnu comme le souligne l'emploi de l'indéfini :

¿De qué te quejas? - me avisa una voz sumergida -. ¿No diste por derribadas las tapias que separan la alucinación de la vigilia ? ¿Pretendías haber recompuesto el estrago simplemente por tomar unas píldoras, comprarte ropa nueva y disfrazarte de chico sano y normal ? Desconfía de ese tipo de milagros. Lo de Gerda fue otra cosa. Ella se lo trabajó, se tiró al mundo y le echó mucha fe. (250)

S'agit-il alors de la voix de la grand-mère qui refait surface bien des années plus tard après son décès ou de celle d'un narrateur extra hétérodiégétique qui renverrait à une sorte de supranarrateur, ou s'agit-il d'une voix propre au narrateur adulte, une voix interne qu'il est le seul à pouvoir entendre? Dans les trois cas de figure, le lien que la voix entretient avec le passé est évident et ce qui est le plus

10 André SIGANOS, Mythe et écriture - La nostalgie de l'archaïque, Paris, PUF écriture, 1999, p. 17. 
surprenant est la possibilité qu'a le héros d'entendre à nouveau cette voix qui appartient au passé, car, comme le dit très justement un autre personnage du roman " $i$ consigue en este momento recordar su voz como si la oyera. Es lo más difícil reproducir una voz en la memoria, y lo más misterioso, ¿verdad?"(p. 52). La voix du conte s'imprègne alors de mystère ; un mystère qui va s'intensifier encore puisque si les voix du conte permettent dans un premier temps de retrouver le passé, il faut aussi remarquer qu'une fois dans ce passé, le narrateur enfant souhaite reprendre le flambeau que lui a transmis le narrateur adulte et à son tour remonter encore plus le temps. L'enfant est alors en quête d'un début du temps, un retour aux origines que suggèrent fortement les verbes « retroceder » et « reingresar » de même que les expressions « navegar hacia atrás » ou « aquellos comienzos » :

- Pero esta noche no, todavía no, abuela - dije, acomodando mi cuerpo a los repliegues de aquella cueva blanda y ancestral -. Déjame que me quede aquí contigo. ¡ $\mathrm{Si}$ pudiera llorar, como antes de venir la Reina de las Nieves! Pero no puedo. ¿Cómo empezaba el cuento ? ¿Cómo era?

Apreté más los ojos secos. Quería retroceder, navegar hacia atrás, hacia aquellos comienzos de verano antiguos, necesitaba reingresar en el reino del «érase que se era ", mediante el rescate de la fórmula exacta de iniciación. Principiaba el estío... (95)

Le texte précise très bien que le narrateur enfant est à la recherche de la clef, cette "formule exacte de l'initiation » qui lui permettra d'atteindre le début des temps. Or, ce début ne renvoie-t-il pas au temps mythique, comme le propose Mircéa Éliade dans son étude sur Le mythe de l'éternel retour? Par ailleurs, le conte rejoindrait ainsi le mythe duquel il n'est pas si éloigné que cela, comme le remarque Michèle Simonsen ${ }^{11}$. De plus, mythe renvoie au muthos grec, c'est à dire à la parle, au récit et à son écriture. Un rapprochement qui n'est pas passé inaperçu à l'étude d'André Siganos dont les travaux ont été regroupés sous le titre de Mythe et écriture - La nostalgie de l'archaïque.

11 Michèle SIMONSEN, op. cit., p. 13-15. 
Les voix qui accompagnent, entourent, puis émergent du narrateur devenu adulte l'amènent à reconstituer son passé avec l'aide de sa mémoire. Le narrateur se met alors à écrire tout ce qu'il a entendu pour ne plus jamais perdre et oublier son passé :

- De verdad, Leonardo, no te entiendo. es como si hubieras perdido la memoria.

- Ya le he dicho antes que, en parte, la he perdido. Y que ahora, gracias a la escritura, la estoy empezando a recuperar, voy poco a poco. Son secuelas de la cárcel. Pero de todas maneras, aunque sea verdad lo que usted dice de que fui yo quien quiso vender la Quinta Blanca... (161)

En fin de compte, l'oralité trouve son aboutissement dans l'écriture. Le destin ultime des mots et des voix qui les ont fait connaître est de finir "plasmadas en los libros, eso es inevitable " (311). Les mots des voix viennent alors trouver leurs échos sur la page des livres. Comme si cette dernière était un véritable mur sur lequel viendraient s'inscrire les mots prononcés oralement. On s'en rend compte lorsque Leonardo dit que les contes oraux qui lui ont été racontés, et notamment celui de la Reine des Neiges, viennent s'inscrire sur le papier comme s'ils sortaient du crayon du narrateur devenu écrivain : «Tal vez esté mirando alternativamente al Kay con antifaz que sale de mi lápiz y a su « Ángel de las despedidas » (226). La rencontre de Leonardo avec Casilda, la nouvelle propriétaire de la Quinta Blanca, dans les dernières pages du roman, est un retour vers le passé - la Quinta Blanca - et surtout une récupération de sa mémoire (314) grâce à l'oralité incarnée par Casilda - nouvelle narratrice de bien d'autres histoires - mais aussi et surtout grâce au contenu de sa valise :

[...] había metido en ella todos sus cuadernos, y ahora comprendía que perderla habría significado perder el rastro de los últimos meses y de todo el tiempo que dormita en el lago helado de la memoria, Aunque también, quizá, soltar lastre. (322)

Il ne reste plus à Leonardo qu'à tourner l'une des pages de ses cahiers pour débuter une nouvelle vie avec une mémoire récupérée. 
Comme nous avons pu nous en rendre compte à travers l'étude de ce roman de Carmen Martín Gaite, La Reina de las Nieves, la voix narrative est omniprésente sous bien des formes : narrateurs homo et hétérodiégétiques, narrateurs dont on ne peut percevoir l'existence qu'à travers la voix d'autres narrateurs et finalement récits dont le thème central est une voix qui est transmise par l'oralité elle-même. Toutes ces voix se superposent et établissent entre elles des liens : résonances, échos, fusions, confusions. Finalement, la voix orale, tout aussi paradoxal que cela puisse paraître, vient s'inscrire sur la page blanche en empruntant deux voix : tout d'abord celle de l'image et de l'illustration, puis celle des souvenirs et de la mémoire.

C'est ce dernier aspect qui semble intéresser de plus en plus d'écrivains espagnols contemporains. Nous avons cité Juan Marsé, mais aussi des romanciers tels qu'Antonio Muñoz Molina, Javier Marías ou Juan José Millás, pour n'en citer que quelques uns, écrivent des romans de et sur la mémoire en accordant une place fondamentale aux aspects narratifs, à la mise en place de polyphonies. Comme le disait dernièrement l'auteur de Beatus Ille lors d'une conférence sur le thème de "Mémoire et Histoire " ${ }^{12}$, l'homme à besoin pour vivre qu'on lui raconte des histoires pour savoir ce qu'il est et pour continuer à vivre ; et c'est pour cela qu'il y aura toujours des conteurs et des narrateurs pour raviver les souvenirs et faire vivre la mémoire.

12 Conférence donnée à la Villa Gillet à Lyon, le jeudi 30 mars 2000 . 